\title{
Sphingolipids as Mediators in the Crosstalk between Microbiota and Intestinal Cells: Implications for Inflammatory Bowel Disease
}

\author{
Phillips-Farfán Bryan, ${ }^{1}$ Carvajal Karla, ${ }^{1}$ Medina-Torres Edgar Alejandro, ${ }^{2}$ \\ Espinosa-Padilla Sara Elva, ${ }^{2}$ Fabrias Gemma, ${ }^{3}$ and Camacho Luz ${ }^{1}$ \\ ${ }^{1}$ Laboratorio de Nutrición Experimental, Instituto Nacional de Pediatría, 04530 Ciudad de México, Mexico \\ ${ }^{2}$ Unidad de Investigación en Inmunodeficiencias, Instituto Nacional de Pediatría, 04530 Ciudad de México, Mexico \\ ${ }^{3}$ Research Unit on Bioactive Molecules, Department of Biomedicinal Chemistry, Institute for Advanced Chemistry of \\ Catalonia (IQAC-CSIC), 08034 Barcelona, Spain
}

Correspondence should be addressed to Camacho Luz; camacho.luz@gmail.com

Received 31 March 2016; Revised 10 June 2016; Accepted 14 July 2016

Academic Editor: José César Rosa Neto

Copyright @ 2016 Phillips-Farfán Bryan et al. This is an open access article distributed under the Creative Commons Attribution License, which permits unrestricted use, distribution, and reproduction in any medium, provided the original work is properly cited.

Inflammatory bowel disease (IBD) describes different illnesses characterized by chronic inflammation of the gastrointestinal tract. Although the pathogenic mechanisms leading to IBD are poorly understood, immune system disturbances likely underlie its development. Sphingolipids (SLs) have been identified as important players and promising therapeutic targets to control inflammation in IBD. Interestingly, it seems that microorganisms of the normal gut microbiota and probiotics are involved in sphingolipid function. However, there is a great need to investigate the role of SLs as intermediates in the crosstalk between intestinal immunity and microorganisms. This review focuses on recent investigations that describe some mechanisms involved in the regulation of cytokine profiles by SLs. We also describe the importance of gut microbiota in providing signaling molecules that favor the communication between resident bacteria and intestinal cells. This, in turn, modulates the immune response in the bowel and likely in other peripheral organs. The potential of SLs and gut microbiota as targets or therapeutic agents for IBD is also discussed.

\section{Introduction}

Inflammatory bowel disease (IBD) is a collection of digestive tract pathologies with chronic inflammation, such as Crohn's disease (CD) and ulcerative colitis (UC). Both conditions usually cause diarrhea, pain, fatigue, and weight loss, among other symptoms. CD causes inflammation in many parts of the gastrointestinal (GI) tract, whereas UC only affects the colon. Currently, the specific etiology of IBD is not well established. However, research in this field points to GI immune system dysfunction as a central pathogenic component [1]. The GI tract is continuously exposed to a great diversity of antigens from foods, bacteria, and parasites. Consequently, immunity in this organ is suppressed to avoid inflammation and maintain homeostasis. Any disruption in the regulatory mechanisms of the GI immune system leads to an excessive response that causes chronic inflammation [2, 3]. Recently, sphingolipids (SLs), particular membrane lipids, have been shown to play an important role in modulating the GI immune response and are promising therapeutic targets for IBD [4]. It has been also observed that the bacteria that naturally colonize the gut, named microbiota, may be involved in the metabolism of SLs, including their biosynthesis. Thus, microbiota and their interaction with probiotics likely are pivotal players in regulating GI immunity. The crosstalk between these components needs to be investigated more deeply. In this review, we will focus on the relationships between SLs, intestinal microbiota, and probiotics with a particular emphasis on their influence upon IBD. 


\section{Sphingolipids}

Sphingolipids (SLs) are plasma membrane components involved in controlling cellular processes such as proliferation, migration, and apoptosis [5]. Moreover, some bacterial species (Bacteroides, Sphingomonas, etc.) are capable of synthesizing these lipids $[6,7]$. SLs are composed of a sphingoid backbone attached to a fatty acid via an amide bond. The main SLs include ceramide (Cer), ceramide-1-phosphate (C1P), glucosylceramide (GC), sphingomyelin (SM), sphingosine (Sph), and sphingosine-1-phosphate (S1P) (Figure 1(a)). S1P is antagonistic to Cer and Sph, since it promotes cell growth and inhibits apoptosis. The enzymes that interconvert Cer, $\mathrm{Sph}$, and S1P regulate their functions. The metabolism of SLs and the participating enzymes is reviewed elsewhere $[8,9]$. However, it is important to mention that Cer has a central role in their metabolism, since it can be synthesized by de novo pathway or derived from complex SLs such as SM $[9,10]$ and glycosphingolipids (Figure 1(b)).

\section{Cer Plays a Harmful Role in IBD}

Cer and related products contribute to varied biological processes as signaling molecules; in addition they are also involved in the development and progression of several human diseases including IBD. The hydrolysis of SM, catalyzed by sphingomyelinases (SMases), is an important source of Cer. However, it can be synthesized by other pathways, which may include the participation of certain microorganisms and cytokines. For instance, the major constituent of the outer membrane of Gram-negative bacteria (lipopolysaccharide or LPS) activates acid SMase in macrophages, which increases Cer content [11,12]. Once produced, Cer and related lipids participate in inflammatory processes of several tissues, where they stimulate immune cells [13-15] by triggering their mitogen-activated protein kinase (MAPK) pathway [16]. The data shows that IL-1 and Cer augment inflammation via increased eicosanoid production $[17,18]$. This possibly contributes to tumor development associated to $\operatorname{IBD}[19,20]$. Table 1 summarizes both the harmful and beneficial effects of Cer.

It is known that interleukin- (IL-) 1 dose- and timedependently increases Cer accumulation in intestinal epithelial cells (IEC) in vitro, increasing the inflammatory response [21]. The way this IL-1-induced Cer rise modulates the immune response is reported. IL-1 or Cer treatment has no effect on IEC, but a cyclooxygenase- (COX-) 2 inhibitor increases their apoptosis. In addition, IL-1 or Cer increases activation of the nuclear factor $\kappa$-light-chain-enhancer of activated B cells $(\mathrm{NF}-\kappa \mathrm{B})$ in a time and dose dependent manner, by reducing the levels of inhibitor of $\kappa$-light-chainenhancer of activated $\mathrm{B}$ cells $\mathrm{I} \kappa \mathrm{B} \alpha$ and $\mathrm{I} \kappa \mathrm{B} \beta$. The effect requires the degradation of $\mathrm{I} \kappa \mathrm{B} \alpha$ and $\mathrm{I} \kappa \mathrm{B} \beta$ by the proteasome [19]. IL-1 or Cer augments the production of the antiapoptotic protein B-cell lymphoma- (BCL-) 2, while reducing the expression of several proapoptotic molecules: BCL-2 associated protein $\mathrm{X}$ (BAX), BCL-2 homologous antagonist/killer (BAK), and BCL-2 associated death promoter (BAD) $[19,20]$. IL-1 or Cer decreases cyclin-dependent kinase inhibitor p21 levels and the number of cells in the $G_{0} / G_{1}$ phase of the cell cycle, while augmenting cells in the $G_{2} / M$ phase. All these data suggest that IL-1 and Cer enhance survival of IEC by activating COX-2 and NF- $\kappa \mathrm{B}$, which results in reduced proapoptotic protein expression and increased levels of antiapoptotic molecules. Thus, augmented inflammation and reduced apoptosis of IEC may contribute to tumorigenesis in IBD patients.

On the other hand, tumor necrosis factor- (TNF-) $\alpha$ and interferon- $\gamma$ induce apoptosis of IEC and also impair their barrier function [21]. These proinflammatory proteins result in Cer production, which may be at least partially responsible for their effects on barrier action. In agreement, exogenous SMase dose-dependently increases IEC permeability in vitro [34]. Consistently, SMase treatment diminishes transepithelial resistance and a Cer antibody blocks the augmented permeability caused by platelet activating factor (PAF). Lipid rafts (detergent-insensitive glycosphingolipid-enriched domains) in epithelial cells show high levels of SM, Cer, and cholesterol, plus the tight-junction proteins occludin and claudin-4. In fact, Cer colocalizes with a tight-junction protein. Incubating IEC with exogenous SMase results in a fast elevation of Cer plus a reduction of SM and cholesterol [34]. Thus, the data suggest that proinflammatory stimuli activate SMases, which hydrolyze SM into Cer. Cer accumulates in junctional complexes, reducing their cholesterol levels and provoking their destabilization, which eventually produces a dysfunctional epithelial barrier in the intestine.

In this sense, incubation of a colon cancer cell line with exogenous SMase results in rapid elevation of the mRNA for matrix metalloproteinase- (MMP-) 1 and MMP-10. In fact SMase dose-dependently increases the expression of MMP1 protein. IL- $1 \beta$ and TNF- $\alpha$ augment MMP-1 production in colon cancer cells and fibroblasts from healthy subjects and patients with UC. MMP-1 degrades the extracellular matrix and is thought to damage the colonic mucosa. Inhibition of acidic SMase with imipramine blocks the effect of IL- $1 \beta$ and TNF- $\alpha$ on MMP-1 [22]. Thus, the results suggest that inhibiting acid SMase activity may be a viable therapeutic option for IBD patients (Table 1).

Accordingly, a SMase inhibitor reduces TNF- $\alpha$, IL- $1 \beta$, and IL-6 LPS-induced release from macrophages and diminishes TNF- $\alpha$ secretion from human peripheral blood mononuclear cells (PBMC) in response to LPS [23]. The inhibitor also decreases TNF- $\alpha$, IL-1 $\beta$, and IL- 6 levels in a colitis animal model produced by administering dextran sulphate sodium (DSS) and reduces the increase in macrophage Cer levels and NF- $\kappa \mathrm{B}$ stimulation caused by LPS. This inhibitor also prevents the increase in macrophage acid SMase activity caused by LPS or TNF- $\alpha$. Finally, SM inhibition also increases the viability of cells incubated in media from macrophages exposed to LPS and decreases colon inflammation [23].

The injury caused by Cer is actually caused by its metabolic products, particularly its phosphorylated forms. The harmful Cer derivatives are produced by key enzymes involved in SL metabolism. In this line, the activity of neutral ceramidase (nCDase), an enzyme that catalyzes Cer breakdown, increases in the epithelial layer of the colon after treatment with DSS [30]. The role of nCDase is clearly 


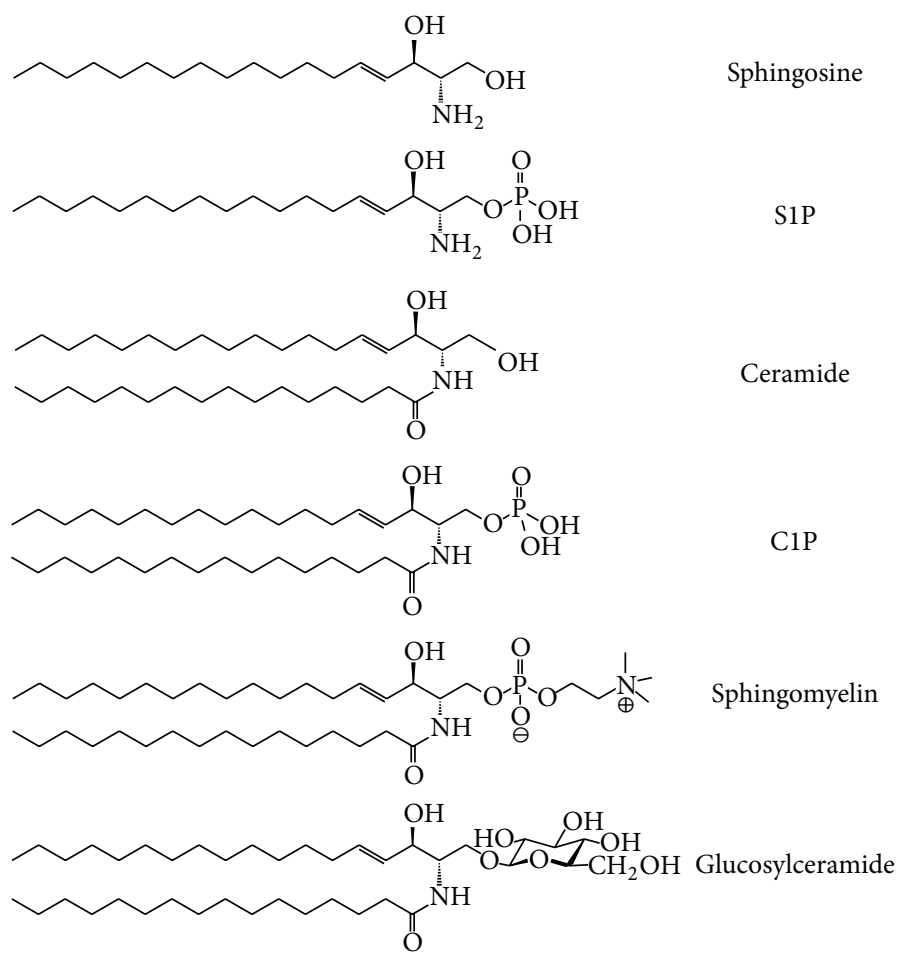

(a)

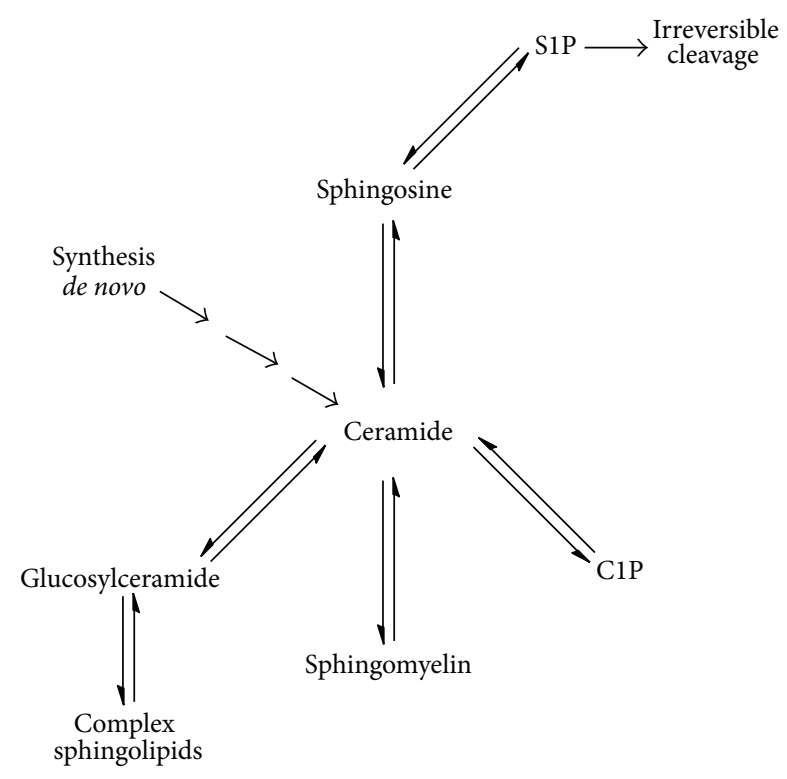

(b)

FIGURE 1: (a) Structure of the main bioactive sphingolipids: sphingosine (Sph), sphingosine-1-phosphate (S1P), ceramide (Cer), ceramide1-phosphate (C1P), sphingomyelin (SM), and glucosylceramide (GluCer). (b) Schematic representation of the central role of ceramide in sphingolipid metabolism.

demonstrated in a null mutant mouse. After DSS treatment, Cer increases in the epithelial layer of the colon in both wildtype and nCDase ${ }^{-/-}$mice, while S1P concentration decreases in wild-type mice but increases in $n \mathrm{CDase}^{-/-}$animals. Cer only increases in the blood of mutant mice due to DSS, while systemic S1P levels augment in both wild-type and nCDase $\mathrm{e}^{-/-}$ mice after DSS administration. TNF- $\alpha$ and COX-2 expression increase after DSS treatment in the epithelial layer of wildtype and mutant mice, respectively. DSS causes systemic inflammation in both genotypes, as indicated by decreased red blood cells and increased white blood cells; though neutrophils and lymphocytes are higher in $\mathrm{nCDase}^{-/-}$mice. Finally, endotoxin levels are increased in the serum of mutant mice after DSS administration. Thus, nCDase may protect against inflammation, since when it is lacking worse UC symptoms develop [30].

Oral SM ingestion increases SM in feces and IEC of DSS-treated mice [24]. SM feeding may be harmful since it increases weight loss, intestinal mucosal inflammation, and epithelial damage in mice exposed to DSS. Inflammation of the intestinal mucosa is also augmented by dietary SM in IL-10 ${ }^{-/-}$mice. Importantly, SM supplementation results in higher cathepsin D activity and IEC apoptosis [24]. SM feeding increases Cer in control and DSS-treated mice [25]. Similarly, a human colon cell line (HT-29) also converts SM into Cer. Cathepsin D and a proapoptotic protein (BCL-2 homology 3 interacting-domain death agonist or BID) augment in
HT-29 cells upon SM treatment. Indeed, SM and DSS activate BID and reduce BCL-2 levels. SM causes apoptosis of HT-29 cells and IEC but phosphatidylcholine protects them. Similarly, SM affects tight-junction proteins making tight junctions weaker, whereas phosphatidylcholine has the opposite effect [25].

\section{Cer Plays a Beneficial Role in IBD}

There are also studies suggesting that Cer and SMases, especially if exogenously applied, may be beneficial for IBD treatment. In this line, it is important to note that their action may depend on how and where Cer is produced. For instance, exogenous acidic and neutral SMases dose-dependently increase Cer levels and trigger NF- $\kappa \mathrm{B}$, mimicking the effect of TNF- $\alpha$ [35]. However, the effect of these SMases on NF- $\kappa$ B activation differs in kinetics and the stimulated $\kappa \mathrm{B}$ complexes. Acidic SMase turns on p50/p50 homodimers later (20 hours) than neutral SMase, which activates RelA/p52 or RelA/p50 heterodimers at 30 minutes. In fact, IL-8 expression is more than double with neutral SMase compared to acidic SMase. Lastly, the latter SMase induces apoptosis of colon cancer cells in vitro but neutral SMase has no effect unless NF- $\kappa \mathrm{B}$ is inactive. Therefore, Cer-induced apoptosis may depend on the enzyme that produces it or the site where it is produced [35].

Alkaline SMase is a nucleotide pyrophosphatase/phosphodiesterase family member that breaks down dietary SM [36]. Interestingly, it hydrolyzes PAF in vitro, while neutral 
TABle 1: Potential harmful and beneficial mechanisms of Cer and S1P in IBD.

\begin{tabular}{|c|c|c|c|c|}
\hline SLs & Effect & Target & Tissue/cells & References \\
\hline \multicolumn{5}{|c|}{ Harmful } \\
\hline \multirow{5}{*}{ Cer } & \multirow{3}{*}{ Increases } & $\mathrm{NF}-\kappa \mathrm{B} \& \mathrm{COX}-2$ & Intestinal tract & {$[12,13]$} \\
\hline & & BCL-2 expression & Intestinal tract & {$[12,13]$} \\
\hline & & Inflammation & Intestinal tract & {$[12,13]$} \\
\hline & \multirow{2}{*}{ Decreases } & Apoptosis & Intestinal tract & {$[12,13]$} \\
\hline & & Expression of BAX, BAK \& BAD & Intestinal tract & {$[12,13]$} \\
\hline \multirow{4}{*}{ Cer } & \multirow{2}{*}{ Activates } & Immune cells & Intestinal tract & {$[14-16]$} \\
\hline & & MAPK cascade & Intestinal tract & {$[17]$} \\
\hline & \multirow{2}{*}{ Decreases } & Cholesterol levels & IEC tight junctions & {$[20,21]$} \\
\hline & & Barrier function & IECs & {$[20,21]$} \\
\hline \multirow{6}{*}{ SMase } & \multirow{6}{*}{ Increases } & TNF- $\alpha$, IL-1 $\beta$ \& IL-6 secretion & Intestinal macrophages & {$[22]$} \\
\hline & & LPS-induced TNF- $\alpha$ release & PBMCs & {$[22]$} \\
\hline & & TNF- $\alpha$, IL-1 $\beta$ \& IL- 6 levels in DSS-induced colitis & Colon & {$[22]$} \\
\hline & & LPS-induced increase of Cer \& NF- $\kappa \mathrm{B}$ & Intestinal macrophages & {$[22]$} \\
\hline & & Inflammation & Colon & {$[22]$} \\
\hline & & LPS-induced cell death & Intestinal tract & {$[22]$} \\
\hline \multirow{2}{*}{ nCDase } & \multirow{2}{*}{ Decreases } & DSS-induced S1P and COX-2 levels & Colon & {$[23]$} \\
\hline & & Endotoxin levels & Serum & {$[23]$} \\
\hline \multirow{3}{*}{ Dietary SM } & \multirow[b]{2}{*}{ Increases } & DSS-induced inflammation & Colon & {$[24,25]$} \\
\hline & & $\begin{array}{l}\text { Cathepsin D activity, BID activation, HT-29 cell \& IEC } \\
\text { apoptosis }\end{array}$ & Colon & {$[24,25]$} \\
\hline & Decreases & BCL-2 levels & Colon & {$[24,25]$} \\
\hline \multicolumn{5}{|c|}{ Beneficial } \\
\hline Cer & Decreases & $\begin{array}{c}\text { IL-6 synthesis } \\
\text { Mast cell degranulation }\end{array}$ & Colon & {$[26]$} \\
\hline \multirow{5}{*}{ Alkaline SMase } & \multirow{4}{*}{ Decreases } & PAF & Intestinal tract & {$[16]$} \\
\hline & & DSS-induced inflammation & Rectum & {$[27]$} \\
\hline & & Tumor incidence & Colon & {$[28]$} \\
\hline & & DSS + azoxymethane cancer aggressiveness & Colon & {$[28]$} \\
\hline & Protects & & Colonic epithelium & {$[29]$} \\
\hline Acidic SMase & Increases & Apoptosis & Colon cancer cells & {$[30]$} \\
\hline \multirow{9}{*}{ Dietary SM } & \multirow{4}{*}{ Decreases } & DSS-induced inflammation & Colon & [29] \\
\hline & & Lymphocyte entry & Colon, PPAR- $\gamma^{-/-}$mice & {$[31]$} \\
\hline & & Carcinoma burden & Colon, PPAR- $\gamma^{-/-}$mice & {$[31]$} \\
\hline & & $\mathrm{F} 4 / 80^{+}$macrophages & Mesenteric lymph node & {$[31]$} \\
\hline & \multirow{2}{*}{$\begin{array}{l}\text { Delays } \\
\text { Hastens }\end{array}$} & Inflammation & Intestinal tract & {$[31]$} \\
\hline & & Recovery & Intestinal tract & {$[31]$} \\
\hline & \multirow{3}{*}{ Increases } & Survival of PPAR- $\gamma^{-/-}$mice & & {$[31]$} \\
\hline & & Chemokines and their receptors & Intestinal tract, PPAR- $\gamma^{-/-}$mice & {$[31]$} \\
\hline & & $\mathrm{CD} 4^{+} \mathrm{T}$ cell maturation genes & Intestinal tract, $\mathrm{PPAR}-\gamma^{-/-}$mice & {$[31]$} \\
\hline \multirow[b]{2}{*}{ S1P } & Increases & B \& T cell survival & Intestinal tract & {$[26]$} \\
\hline & Decreases & $\begin{array}{l}\text { T cell proliferation } \\
\text { Cytokine synthesis }\end{array}$ & Intestinal tract & {$[26]$} \\
\hline S1P & Increases & COX-2 \& $\mathrm{PGE}_{2}$, inflammation, metalloproteinase production & Intestinal tract & {$[32,33]$} \\
\hline
\end{tabular}


SMase does not affect PAF [15]. In fact, alkaline SMase activity against SM or PAF is inhibited by high amounts of PAF or $\mathrm{SM}$, respectively. Moreover, its effect on PAF hydrolysis is dose- and time-dependent and is enhanced by bile salts. Low concentrations of zinc $(0.1-0.25 \mathrm{mM})$ stimulate its activity against PAF, while higher levels dose-dependently inhibit PAF hydrolysis. Importantly, PAF incubation with alkaline SMase eliminates its functional effects: p42 and p44 MAPK phosphorylation, IL-8 release, and leukocyte chemotaxis are inhibited [15]. Since PAF displays proinflammatory effects $[29,37]$, these results suggest that alkaline SMase plays a protective role against the development of IBD and colon cancer. In fact, two reports show that this is indeed the case. Rectal administration of alkaline SMase diminishes DSS-induced inflammation and preserves the colonic epithelium [27]. Similarly, mice lacking alkaline SMase show higher colon tumor incidence and more aggressive cancers due to azoxymethane plus DSS treatment [28].

Oral SM administration may reduce inflammation caused by DSS [31]. Similarly, dietary SLs block tumor development and repress colon cancer [38, 39]. In line, inflammation onset is delayed by SM feeding in mice lacking peroxisome proliferator-activated receptor- (PPAR-) $\gamma$ in epithelial and hematopoietic cells and their recovery is accelerated [40]. Dietary SM increases survival, reduces harmful colonic changes, and diminishes tumor area in PPAR- $\gamma^{-/-}$mice. SM supplementation also lowers lymphocyte infiltration into the colon, reduces carcinoma load, decreases F4/80 macrophages in the mesenteric lymph node, and tends to reduce cluster of differentiation (CD) $4^{+} \mathrm{T}$ cells in both mutant and wild-type mice. SM feeding augments several chemokines plus their receptors and genes that participate in the differentiation of $\mathrm{CD} 4^{+} \mathrm{T}$ cells towards both proinflammatory and anti-inflammatory phenotypes. Dietary SM is anti-inflammatory by reducing regulatory gene expression and modifying genes involved in tissue protection or regeneration, suggesting that SM feeding may alter tumor development by reducing inflammation [40]. SM may affect inflammatory processes in a PPAR- $\gamma$ dependent manner but its effect on cancer seems independent of this receptor. Thus, SM ingestion may be beneficial or harmful (see above), perhaps depending on its source.

The extracellular action of Cer has shown beneficial effects in the damaged colon, maybe by binding to receptors not related to SLs, such as the leukocyte monoimmunoglobulin-like receptor 3 (LMIR3) [41]. LMR3 ${ }^{-/-}$mice are very vulnerable to colitis induced by DSS, shown by increased weight loss and disease activity as well as reduced colon length and survival [26]. These mutants show greater infiltration of neutrophils, eosinophils, and mononuclear cells into the colon. These cells together with mast cells express LMIR3 on their surface and DSS treatment increases its expression in mast cells. DSS augments the number of mast cells in both genotypes, but this is greater in LMIR3 ${ }^{-/-}$mice. Similarly, degranulated mast cells are also higher in mutant mice. IL-6, IL-17A, and TNF- $\alpha$ as well as chemokine transcripts and proteins are increased by treatment with DSS in LMIR3 ${ }^{-/-}$mice. Bone marrow and mast cell transplantation show that the latter cells participate in colitis aggravation in mutant mice. Injury to the colon results in the presence of extracellular ATP which activates P2X7 purinoceptors in mast cells, which release inflammatory molecules [42]. Consistent with this, DSS increases the levels of ATP in the colon. In the absence of Cer, ATP treatment increases mast cell degranulation and secretion of neutrophil chemoattractants such as leukotriene B4, while Cer represses these effects in wild-type but not LMIR3 $^{-/-}$mice. Similarly, Cer inhibits IL- 6 synthesis in wildtype mast cells but not in mast cells from mutant mice. Anti-Cer antibodies worsen colitis symptoms in wild-type but not LMIR3 ${ }^{-/-}$mice, while Cer liposomes suppress mast cell degranulation in the colon of wild-type mice but not in mutant mice [26]. The data suggest that Cer liposomes may actually be useful as an IBD therapeutic strategy.

\section{Sphingosine-1-Phosphate and IBD}

S1P is synthesized from Sph, a product of Cer breakdown, by two enzymes: sphingosine kinase 1 and sphingosine kinase 2 (SK1 and SK2). Once produced, S1P exerts its action by two mechanisms: directly via intracellular targets or by binding to one of its five different membrane receptors named S1PRs [43]. S1P degradation is regulated reversibly by S1P phosphatases or irreversibly by the S1P lyase enzyme (S1PL). S1P has a significant role in regulating immune cell trafficking, inflammation, angiogenesis, and enhancing cell survival. S1P treatment enhances the survival of B and T cells and inhibits both homoeostatic proliferation and $\mathrm{T}$ cell receptorinduced proliferation of T cells, as well as inhibiting cytokine production [44].

It was proposed that S1P favors cell proliferation and survival, as well as inflammation mediated by prostaglandins since it acts as a chemoattractant agent for basophils, neutrophils, and $\mathrm{NK}$ cells by upregulating COX-2 and $\mathrm{PGE}_{2}$ expression $[32,33,45,46]$. It is important to mention that the inflammatory response is not limited to the effects of S1P and $\mathrm{C} 1 \mathrm{P}$ expression in the intestinal tissue. The resulting $\mathrm{PGE}_{2}$ expression can induce the production of interleukins 4,5 , and 10 (Th2 profile) and negatively regulate the expression of interferon $\gamma$, TNF- $\alpha$, and interleukins $1 \beta, 2$, and 12 [47]. Th2 profile cytokines can then induce further expression of $\mathrm{PGE}_{2}$ and COX-2 in the intestinal tissue, which sustains and increases inflammation. The rise in prostaglandin and COX2 induces metalloproteinase expression, which, due to the inflammatory conditions that predominate in the intestinal tissue, favor its destruction (Table 1). The principal effect of inflammation on the intestine is the loss of function and structure of the intestinal mucosa and as a consequence failure in the absorption of nutrients, translocation of microbiota bacteria, and changes in the intestinal microenvironment that favor the development of pathogenic bacteria. Thus, S1P has a harmful role in inflammatory illnesses including IBD [48].

Modulating S1P signaling has been proposed as a therapeutic target in IBD treatment [49]. In this line, several studies have been published which use S1PR antagonists in animal models of colitis. Two independent reports show that treatment with FTY20 significantly attenuates the development 


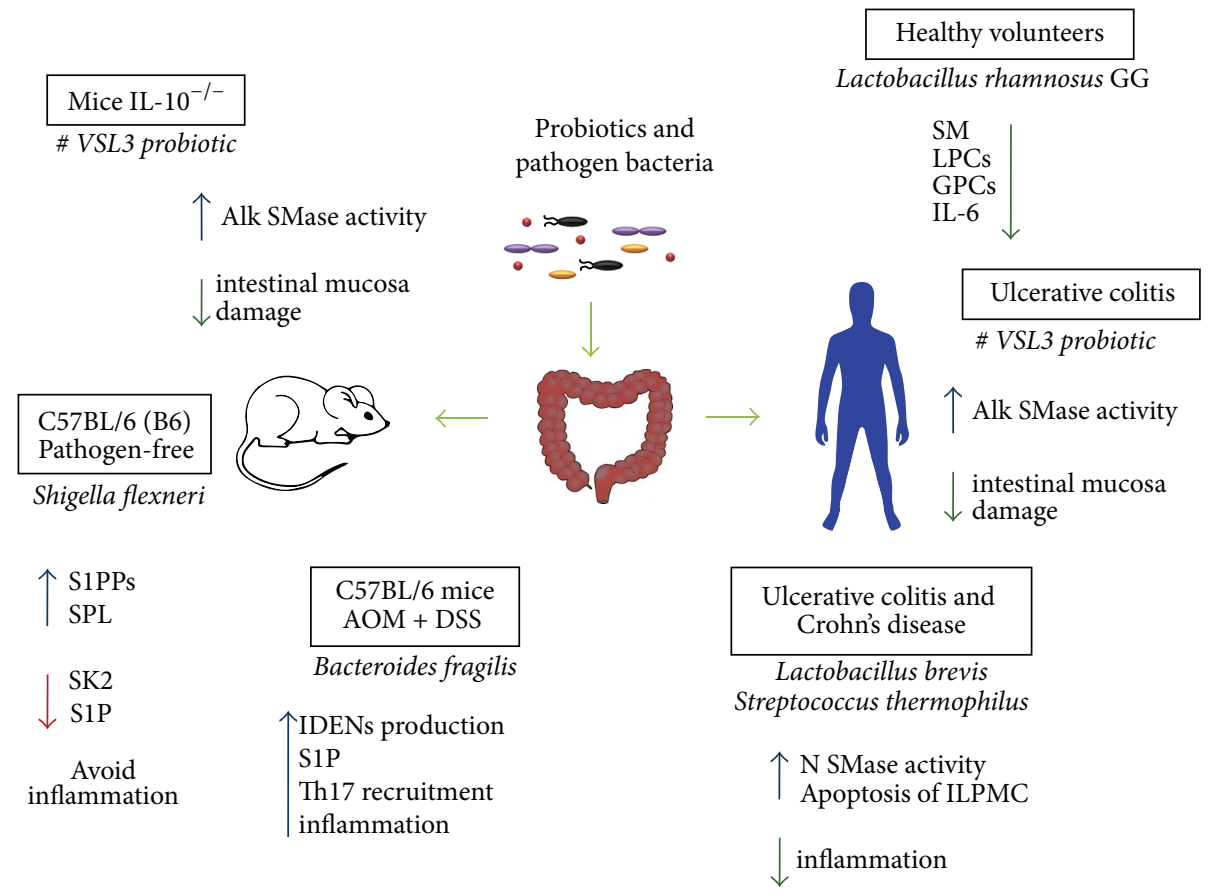

FIGURE 2: Known effects of pathogenic bacteria, microbiota members, and probiotics on SL signaling in IBD. Probiotics increase SMase activity and diminish intestinal inflammation reducing mucosal damage in both humans and a mouse model. Bacteroides fragilis, a known microbiota member, induces inflammation by stimulating epithelial production of IDENs containing high levels of S1P and mediating Th17 recruitment. Conversely, the pathogen Shigella flexneri can avoid the inflammatory response by decreasing S1P levels, downregulating SK2 expression and increasing SPL and S1PPs expression. SM: sphingomyelin, LPCs: lysophosphatidylcholines, GPCs: glycerophosphatidylcholines, IL-6: interleukin-6, ILPMC: intestinal lamina propria mononuclear cells, IDENs: intestinal derived exosome like nanoparticles, S1P: sphingosine 1 phosphate, SK2: sphingosine 1 phosphate kinase 2, SPL: sphingosine 1 phosphate lyase, and S1PPs: sphingosine 1 phosphate phosphatases.

of colitis induced by DSS or due to genetic deficiency of IL-10. In addition, similar results are obtained with the use of two different antagonists: W-061 and KRP-203 [50-53]. On the other hand, SK1 knockout mice are less susceptible of developing colitis after treatment with DSS [54]. Similarly, administration of SK inhibitors (ABC747080 and ABC294640) reduces the development of colitis induced by DSS in a mice model [55]. Additionally, treatment with a selective S1P1 receptor agonist (SEW2871) improves colitis symptoms in IL-10 deficient mice [56].

\section{Probiotics Exert Beneficial Actions by Modifying Intestinal Lipids}

Our bodies are colonized by trillions of microorganisms from more than 1000 different species. The majority of microbes colonizes the gut, having an important role in nutrition and may be associated with bowel diseases [57]. It has been proposed that dysbiosis (an imbalance in the quantity or type of gut microorganisms) produces or worsens inflammatory diseases. In this sense, diverse therapies using probiotics have been proposed as treatments for IBD [58]. Probiotics are defined as living microorganisms which, when administered in adequate amounts, confer a health benefit on the host [59].

Probiotics show immunomodulatory actions in vitro, in animal models and in humans $[60,61]$, especially in the context of inflammatory diseases [62]. In this line, treatment with Lactobacillus rhamnosus GG causes significant lipid alterations in healthy humans [63]. This treatment increases triacylglycerols but decreases lysophosphatidylcholines (LPCs), glycerophosphatidylcholines, and SMs. These changes correlate moderately with IL-6 levels, especially the diminished LPCs, and may participate in the beneficial effects of this probiotic on intestinal epithelial barrier function [63] as illustrated in Figure 2.

Similarly, a probiotic with eight different bacterial strains has beneficial effects on the intestine [64]. IL-10 knockout mice have lower baseline activity of alkaline SMase compared to wild-type animals and treatment with this probiotic augments its function in the ileum and colon [65]. The probiotic increases alkaline SMase action in wild-type mice and reduces damage to the colonic mucosa in IL-10 knockouts. In humans suffering from UC, the probiotic elevates alkaline SMase function and diminishes disease activity [65]. Thus, the results suggest that augmented activity of alkaline SMase is beneficial in patients with IBD.

Sonicates of probiotic Lactobacillus brevis or Streptococcus thermophilus show high neutral SMase activity compared to sonicates of nonprobiotic Escherichia coli and Escherichia faecalis [66]. L. brevis sonicates cause apoptosis of intestinal lamina propria mononuclear cells (ILPMC) from healthy individuals and patients with UC or CD. Interestingly, the effect 
is more pronounced in the latter. Similarly, sonicates from S. thermophilus result in elevated apoptosis of ILPMC from people suffering IBD. Activation of PBMC and ILPMC with antibodies for CD3 and CD28 results in higher apoptosis of these cells in response to the probiotic organisms. Exogenous Cer or neutral SMase also augment apoptosis of ILPMC from healthy subjects and individuals with IBD (Figure 2). Again, the effect is less evident in the former. Both $L$. brevis sonicates and exogenous neutral SMase increase c-jun $\mathrm{N}$-terminal kinase activation in ILPMC. L. brevis sonicates cause the production of higher levels of reactive oxygen species in ILPMC. Sonicates of L. brevis and S. thermophilus incubated with glutathione lose their ability to induce apoptosis of ILPMC from healthy individuals and those affected with CD. In a similar fashion, glutathione abrogates the apoptotic effect of the sonicates on activated PBMC. A specific inhibitor of neutral SMase diminishes apoptosis of ILPMC induced by L. brevis sonicates [66]. These results suggest that probiotics generate elevated levels of Cer via their endogenous SMase, which exerts anti-inflammatory effects by killing resident and blood-derived immune cells.

Although the exact mechanism is not completely identified, some bacteria can modulate the host immune system by modulating S1P levels. For example, a study showed that, in a mouse model and a human cell line, Shigella flexneri downregulates SK2 expression but upregulates the expression of sphingosine 1 phosphate lyase (SPL) and sphingosine 1 phosphate phosphatases (S1PPs), thus decreasing S1P levels as a mechanism to avoid the inflammatory response [67]. Conversely, secreted particles from enterotoxigenic Bacteroides fragilis stimulate intestinal epithelial cells to produce intestinal derived exosome like nanoparticles (IDENs). These IDENs contain elevated levels of S1P, CCL20, and $\mathrm{PGE}_{2}$, which mediate Th17 recruitment and induction contributing to intestinal inflammation and cancer [68] (Figure 2).

\section{Invariant Natural Killer T Cells and Bacterial $\alpha$-Galactosylceramide}

Probiotic bacteria may produce compounds quite similar to $\alpha$-galactosylceramide, which potently activates invariant natural killer T cells (iNKT). These cells are specifically reduced by treatment with azoxymethane followed by DSS, which induces colitis and colon cancer [69]. Mice lacking iNKT cells show more and bigger tumors, as well as worse inflammation indicators than wild-type mice. Azoxymethane/DSS administration augments $\mathrm{CD} 25^{+} \mathrm{CD} 4^{+} \mathrm{T}$ cells and $\mathrm{NK} 1.1^{+}$ $\mathrm{T}$ cells in mice lacking iNKT cells. The number of IL-13 ${ }^{+}$ $\mathrm{CD}^{+}$cells and IL-13 release in the mesenteric lymph node and colon are increased in these animals. Importantly, $\alpha$ galactosylceramide administration elevates the Th1/Th2 ratio in the mesenteric lymph node, while inflammation and the number of tumors are decreased in the colon. Treatment with $\alpha$-galactosylceramide diminishes the number of colonic NK1.1 $1^{+} \mathrm{T}$ cells and IL-13 release from colonic lymphocytes [69]. The data suggest that probiotics stimulate iNKT cells, which reduce inflammation and development of colon cancer by altering $\mathrm{T}$ cell populations and cytokine secretion.
An investigation of the SLPs synthesized by a prominent constituent of the gut microbiota was performed [70]. A putative serine palmitoyltransferase, which catalyzes the first committed step of sphingolipid biosynthesis, was deleted from Bacteroides fragilis NCTC 9343. No double mutants are obtained, suggesting that the enzyme is critical for survival. Single mutants lack sphingolipid production, allowing identification of the sphingolipids produced by wildtype $B$. fragilis. These bacteria synthesize Cer phosphorylethanolamine, its matching dihydroceramide analog, and $\alpha$-galactosylceramide. The latter binds to CD1d to activate mouse and human iNKT cells, as shown by IL- 2 and IFN- $\gamma$ synthesis. This lipid also stimulates human PBMC, as shown by proliferation of $\mathrm{CD}^{+} \mathrm{V} \alpha 24^{+}$cells, and activated iNKT cells in vivo, evidenced by higher CD25, CD69, and IFN- $\gamma$ expression (Figure 3). Moreover the effect is blocked by CD1d antibodies [70]. The results suggest that an important member of the gut microbiota produces $\alpha$-galactosylceramide, which stimulates PBMC and iNKT cells.

Another study suggested that $\alpha$-galactosylceramide did not activate iNKT cells, but instead it reduced their stimulation both in vitro and in vivo by competing for CD1d binding or impeding detection by iNKT cells [71]. Mice monocolonized with $B$. fragilis that lack their presumed serine palmitoyltransferase have a higher number of colonic iNKT cells and worse colitis symptoms, evidenced by a more pronounced weight loss as well as higher IL-4 and IL-13 secretion. The iNKT cell population is reduced by neonatal treatment with a CD1d antibody; this blocks oxazoloneinduced colitis in adult mice with altered $B$. fragilis. These animals have similar colonic bacteria number, comparable chemokine levels, analogous iNKT cell stimulation, and apoptosis plus similar polysaccharide A expression. The bacterial SLs diminish iNKT proliferation, but only when mice were exposed to them prenatally. Ceramides, glycosylceramides, and phosphoethanolamine ceramides are present in wild-type but not in mutant $B$. fragilis. iNKT cell activation is decreased by glycosylceramides, including $\alpha$-galactosylceramide. Importantly, $\alpha$-galactosylceramide treatment during the neonatal period diminishes the number of colonic iNKT cells and improves oxazolone-induced colitis in mice with altered bacteria [71]. Thus, $\alpha$-galactosylceramide produced by a prominent constituent of the gut microbiota is beneficial. The issue of whether this lipid stimulates or inhibits iNKT cells remains to be resolved.

Glycosylceramides contained in mammalian milk and serum activate iNKT cells; indeed human iNKT cells are stimulated by cow and human milk [72]. But glycosylceramides from the spleen of Gaucher's disease patient do not affect iNKT cells. This suggests that an unknown ingredient in mammalian milk and serum is responsible for the observed effect because the glycoceramides within cow's milk and Gaucher's spleen are comparable. Upon further analysis, the ingredient is likely $\alpha$-galactosylceramide which is also present in the thymus of mammals and activated iNKT cells [72]. The most probable explanation is that mammalian microbiota is responsible for producing this $\alpha$-galactosylceramide, although the intriguing possibility that it can be synthesized by mammals cannot be dismissed. Of note, in this study 


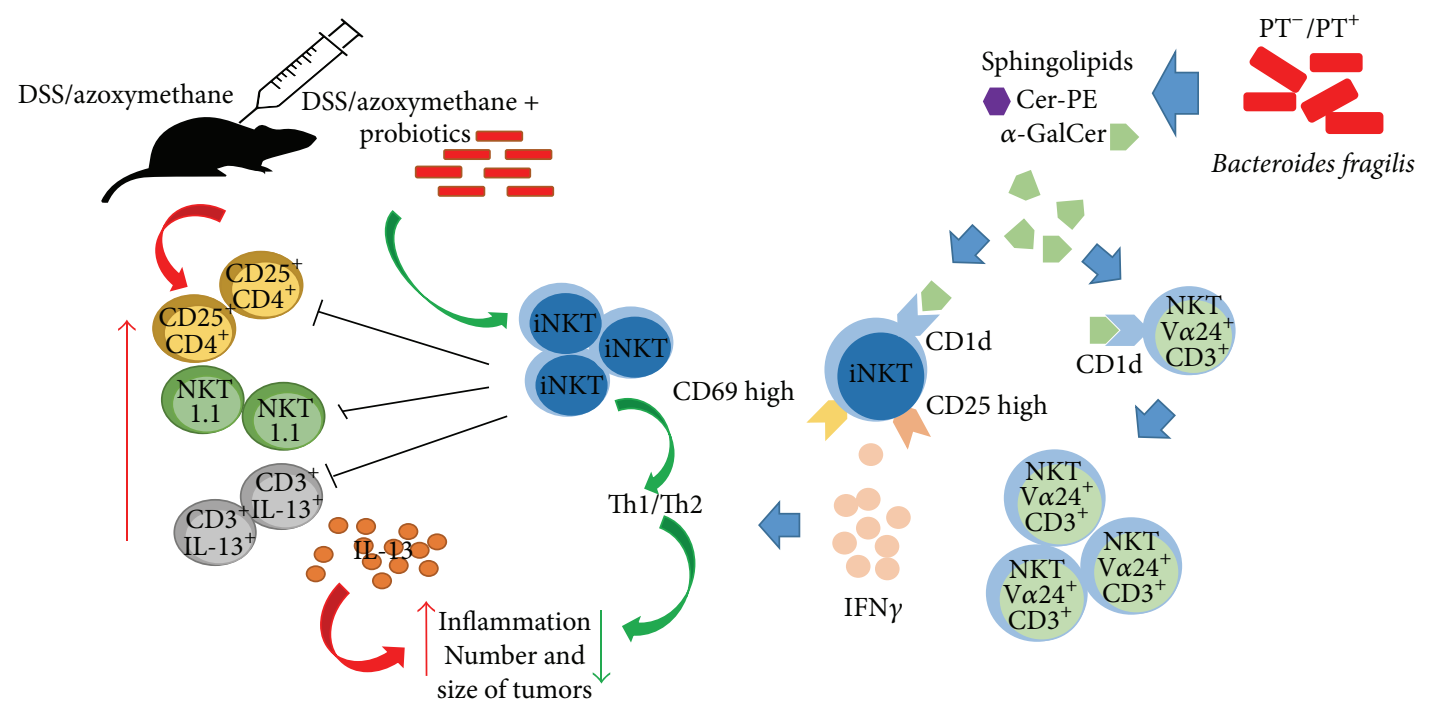

FIGURE 3: Bacterial $\alpha$-galactosylceramide as iNTK regulator in colon cancer. Treatment with azoxymethane followed by DSS reduces the number of iNTK cells and augments $\mathrm{CD} 25^{+} \mathrm{CD} 4^{+} \mathrm{T}$ cells, NKT1.1 ${ }^{+} \mathrm{T}$ cells, and IL- $13^{+} \mathrm{CD} 3^{+}$cells, as well as IL-13 release. All this contributes to form a greater number of tumors of a larger size. Probiotic bacteria can produce compounds similar to $\alpha$-galactosylceramide, which prevents inflammation and reduces the number and size of tumors. $B$. fragilis produces $\alpha$-galactosylceramide which stimulates iNKT cells binding to CD1d, increasing production of IFN- $\gamma$ and proliferation of $\mathrm{CD}^{+} \mathrm{V} \alpha 24^{+}$cells. DSS: dextran sulphate sodium, iNKT: invariant natural killer, NKT: natural killer T cell, and PT: serine palmitoyltransferase.

galactosylceramides stimulate iNKT cells as explained in Figure 3.

\section{Conclusion}

IBD has become an emergent public health problem. As discussed previously, although its exact etiology remains unclear, the role of SLs in contributing to the inflammatory process is evident. Indeed, besides its role in digestive function, the gut is actually considered as an immune organ. This is in large part due to the signaling molecules that are produced within it, particularly those comprised by SLs, which affect not only the intestinal tract but the whole immune system. As mentioned above, Cer is a central molecule in defining the role of the immune response through its different metabolic byproducts: SM, Sph, and S1P, which mediate specific responses. SMases are of particular interest because of their differential activities. Thus, research on therapeutic agents able to modulate SMases and formulations for tissuespecific delivery is mandatory. The conversion of Cer into different SLs, which enhance or prevent the inflammatory response, depends on cell molecular signals and the cell microenvironment. In this sense, gut microbiota may provide the conditions that define the source and fate of SLs as modulators of the immune response. For instance, microorganisms provide enzymes or their activators to promote Cer metabolism and thereby regulate cytokine production. Thus, gut microbiota is a new and attractive target for the control of IBD and even other inflammatory conditions.

In this sense, prebiotic and probiotic agents emerge as pivotal players in the control of the immune response in this organ. Further therapies may include the preservation of natural microbiota in the bowel in order to avoid IBD and new therapies based on nutritional programs including food that favors beneficial species or even seeding the intestinal tract with strains that can produce specific SLs, depending on the pathological situation. These promising possibilities deserve further scientific and clinical investigation.

\section{Competing Interests}

The authors declare that there are no competing interests regarding the publication of this paper.

\section{References}

[1] M. Boirivant and A. Cossu, "Inflammatory bowel disease," Oral Diseases, vol. 18, no. 1, pp. 1-15, 2012.

[2] R. J. Xavier and D. K. Podolsky, "Unravelling the pathogenesis of inflammatory bowel disease," Nature, vol. 448, no. 7152, pp. 427-434, 2007. 
[3] J. Pedersen, M. Coskun, C. Soendergaard, M. Salem, and O. H. Nielsen, "Inflammatory pathways of importance for management of inflammatory bowel disease," World Journal of Gastroenterology, vol. 20, no. 1, pp. 64-77, 2014.

[4] R.-D. Duan and Å. Nilsson, "Metabolism of sphingolipids in the gut and its relation to inflammation and cancer development," Progress in Lipid Research, vol. 48, no. 1, pp. 62-72, 2009.

[5] A. H. Merrill, Biochemistry of Lipids, Lipoproteins and Membranes, Elsevier, 2008.

[6] I. Olsen and E. Jantzen, "Sphingolipids in bacteria and fungi," Anaerobe, vol. 7, no. 2, pp. 103-112, 2001.

[7] D. Ana, C. Na, J. Bielawski, Y. A. Hannun, and D. L. Kasper, "Membrane sphingolipids as essential molecular signals for Bacteroides survival in the intestine," Proceedings of the National Academy of Sciences of the United States of America, vol. 108, no. 1, pp. 4666-4671, 2011.

[8] Y. A. Hannun and L. M. Obeid, "Principles of bioactive lipid signalling: lessons from sphingolipids," Nature Reviews Molecular Cell Biology, vol. 9, no. 2, pp. 139-150, 2008.

[9] M. Maceyka and S. Spiegel, "Sphingolipid metabolites in inflammatory disease," Nature, vol. 510, no. 7503, pp. 58-67, 2014.

[10] P. Gangoiti, L. Camacho, L. Arana et al., "Control of metabolism and signaling of simple bioactive sphingolipids: implications in disease," Progress in Lipid Research, vol. 49, no. 4, pp. 316-334, 2010.

[11] M. L. MacKichan and A. L. DeFranco, "Role of ceramide in lipopolysaccharide (LPS)-induced signaling. LPS increases ceramide rather than acting as a structural homolog," The Journal of Biological Chemistry, vol. 274, no. 3, pp. 1767-1775, 1999.

[12] J. Kornhuber, C. Rhein, C. P. Müller, and C. Mühle, "Secretory sphingomyelinase in health and disease," Biological Chemistry, vol. 396, no. 6-7, pp. 707-736, 2015.

[13] K. J. Claycombe, D. Wu, M. Nikolova-Karakashian et al., "Ceramide mediates age-associated increase in macrophage cyclooxygenase-2 expression," The Journal of Biological Chemistry, vol. 277, no. 34, pp. 30784-30791, 2002.

[14] V. Teichgräber, M. Ulrich, N. Endlich et al., "Ceramide accumulation mediates inflammation, cell death and infection susceptibility in cystic fibrosis," Nature Medicine, vol. 14, no. 4, pp. 382-391, 2008.

[15] J. Wu, Å. Nilsson, B. A. G. Jönsson et al., "Intestinal alkaline sphingomyelinase hydrolyses and inactivates platelet-activating factor by a phospholipase C activity," Biochemical Journal, vol. 394, no. 1, pp. 299-308, 2006.

[16] S. Spiegel, D. Foster, and R. Kolesnick, "Signal transduction through lipid second messengers," Current Opinion in Cell Biology, vol. 8, no. 2, pp. 159-167, 1996.

[17] M. Tsujii and R. N. DuBois, "Alterations in cellular adhesion and apoptosis in epithelial cells overexpressing prostaglandin endoperoxide synthase 2," Cell, vol. 83, no. 3, pp. 493-501, 1995.

[18] F. R. Homaidan, I. Chakroun, G. S. Dbaibo, W. El-Assaad, and M. E. El-Sabban, "IL-1 activates two phospholipid signaling pathways in intestinal epithelial cells," Inflammation Research, vol. 50, no. 7, pp. 375-381, 2001.

[19] F. R. Homaidan, I. Chakroun, and M. E. El-Sabban, "Regulation of nuclear factor- $\kappa \mathrm{B}$ in intestinal epithelial cells in a cell model of inflammation," Mediators of Inflammation, vol. 12, no. 5, pp. 277-283, 2003.

[20] F. R. Homaidan, M. E. El-Sabban, I. Chakroun, M. El-Sibai, and G. S. Dbaibo, "IL-1 stimulates ceramide accumulation without inducing apoptosis in intestinal epithelial cells," Mediators of Inflammation, vol. 11, no. 1, pp. 39-45, 2002.

[21] M. Bruewer, A. Luegering, T. Kucharzik et al., "Proinflammatory cytokines disrupt epithelial barrier function by apoptosisindependent mechanisms," The Journal of Immunology, vol. 171, no. 11, pp. 6164-6172, 2003.

[22] J. Bauer, G. Liebisch, C. Hofmann et al., "Lipid alterations in experimental murine colitis: role of ceramide and imipramine for matrix metalloproteinase-1 expression," PLoS ONE, vol. 4, no. 9, Article ID e7197, 2009.

[23] A. Sakata, K. Yasuda, T. Ochiai et al., "Inhibition of lipopolysaccharide-induced release of interleukin- 8 from intestinal epithelial cells by SMA, a novel inhibitor of sphingomyelinase and its therapeutic effect on dextran sulphate sodium-induced colitis in mice," Cellular Immunology, vol. 245, no. 1, pp. 24-31, 2007.

[24] A. Fischbeck, K. Leucht, I. Frey-Wagner et al., "Sphingomyelin induces cathepsin D-mediated apoptosis in intestinal epithelial cells and increases inflammation in DSS colitis," Gut, vol. 60, no. 1, pp. 55-65, 2011.

[25] K. Leucht, A. Fischbeck, M. Caj et al., "Sphingomyelin and phosphatidylcholine contrarily affect the induction of apoptosis in intestinal epithelial cells," Molecular Nutrition and Food Research, vol. 58, no. 4, pp. 782-798, 2014.

[26] T. Matsukawa, K. Izawa, M. Isobe et al., "Ceramide-CD300f binding suppresses experimental colitis by inhibiting ATPmediated mast cell activation," Gut, vol. 65 , no. 5, pp. 777-787, 2016.

[27] D. Andersson, K. Kotarsky, J. Wu, W. Agace, and R.-D. Duan, "Expression of alkaline sphingomyelinase in yeast cells and antiinflammatory effects of the expressed enzyme in a rat colitis model," Digestive Diseases and Sciences, vol. 54, no. 7, pp. 1440$1448,2009$.

[28] Y. Chen, S.-C. Xu, and R.-D. Duan, "Mevalonate inhibits acid sphingomyelinase activity, increases sphingomyelin levels and inhibits cell proliferation of HepG2 and Caco-2 cells," Lipids in Health and Disease, vol. 14, no. 1, article 130, 2015.

[29] C. C. Yost, A. S. Weyrich, and G. A. Zimmerman, "The platelet activating factor (PAF) signaling cascade in systemic inflammatory responses," Biochimie, vol. 92, no. 6, pp. 692-697, 2010.

[30] A. J. Snider, B. X. Wu, R. W. Jenkins et al., "Loss of neutral ceramidase increases inflammation in a mouse model of inflammatory bowel disease," Prostaglandins and Other Lipid Mediators, vol. 99, no. 3-4, pp. 124-130, 2012.

[31] H. Furuya, S. Ohkawara, K. Nagashima, N. Asanuma, and T. Hino, "Dietary sphingomyelin alleviates experimental inflammatory bowel disease in mice," International Journal for Vitamin and Nutrition Research, vol. 78, no. 1, pp. 41-48, 2008.

[32] G. Bernardini, G. Sciumè, and A. Santoni, "Differential chemotactic receptor requirements for NK cell subset trafficking into bone marrow," Frontiers in Immunology, vol. 4, article 12, 2013.

[33] P. Keul, S. Lucke, K. Von Wnuck Lipinski et al., "Sphingosine-1-phosphate receptor 3 promotes recruitment of monocyte/macrophages in inflammation and atherosclerosis," Circulation Research, vol. 108, no. 3, pp. 314-323, 2011.

[34] J. Bock, G. Liebisch, J. Schweimer, G. Schmitz, and G. Rogler, "Exogenous sphingomyelinase causes impaired intestinal epithelial barrier function," World Journal of Gastroenterology, vol. 13, no. 39, pp. 5217-5225, 2007.

[35] A. Colell, O. Coll, M. Marí, J. C. Fernández-Checa, and C. García-Ruiz, "Divergent role of ceramide generated by exogenous sphingomyelinases on NF- $\kappa \mathrm{B}$ activation and apoptosis in 
human colon HT-29 cells," FEBS Letters, vol. 526, no. 1-3, pp. 15-20, 2002.

[36] R.-D. Duan, Y. Cheng, G. Hansen et al., "Purification, localization, and expression of human intestinal alkaline sphingomyelinase," The Journal of Lipid Research, vol. 44, no. 6, pp. 1241-1250, 2003.

[37] W. Chao and M. S. Olson, "Platelet-activating factor: receptors and signal transduction," Biochemical Journal, vol. 292, part 3, pp. 617-629, 1993.

[38] L. A. Lemonnier, D. L. Dillehay, M. J. Vespremi, J. Abrams, E. Brody, and E. M. Schmelz, "Sphingomyelin in the suppression of colon tumors: prevention versus intervention," Archives of Biochemistry and Biophysics, vol. 419, no. 2, pp. 129-138, 2003.

[39] H. Symolon, E. M. Schmelz, D. L. Dillehay, and A. H. Merrill Jr., "Dietary soy sphingolipids suppress tumorigenesis and gene expression in 1,2-dimethylhydrazine-treated CF1 Mice and ApcMin/+ mice," Journal of Nutrition, vol. 134, no. 5, pp. 1157$1161,2004$.

[40] J. C. Mazzei, H. Zhou, B. P. Brayfield, R. Hontecillas, J. Bassaganya-Riera, and E. M. Schmelz, "Suppression of intestinal inflammation and inflammation-driven colon cancer in mice by dietary sphingomyelin: importance of peroxisome proliferatoractivated receptor $\gamma$ expression," Journal of Nutritional Biochemistry, vol. 22, no. 12, pp. 1160-1171, 2011.

[41] K. Izawa, M. Isobe, T. Matsukawa et al., "Sphingomyelin and ceramide are physiological ligands for human LMIR3/CD300f, inhibiting FceRI-mediated mast cell activation," Journal of Allergy and Clinical Immunology, vol. 133, no. 1, pp. 270-273.e7, 2014.

[42] Y. Kurashima, T. Amiya, T. Nochi et al., "Extracellular ATP mediates mast cell-dependent intestinal inflammation through P2X7 purinoceptors," Nature Communications, vol. 3, article 1034, 2012.

[43] P. J. Gonzalez-Cabrera, S. Brown, S. M. Studer, and H. Rosen, "S1P signaling: new therapies and opportunities," F1000Prime Reports, vol. 6, article 109, 2014.

[44] J. Rivera, R. L. Proia, and A. Olivera, "The alliance of sphingosine-1-phosphate and its receptors in immunity," Nature Reviews Immunology, vol. 8, no. 10, pp. 753-763, 2008.

[45] M. M. Rahman, H. Alkhouri, F. Tang, W. Che, Q. Ge, and A. J. Ammit, "Sphingosine 1-phosphate induces neutrophil chemoattractant IL-8: repression by steroids," PLoS ONE, vol. 9, no. 3, Article ID e92466, 2014.

[46] L. R. Ballou, C. P. Chao, M. A. Holness, S. C. Barker, and R. Raghow, "Interleukin-1-mediated PGE2 production and sphingomyelin metabolism. Evidence for the regulation of cyclooxygenase gene expression by sphingosine and ceramide," The Journal of Biological Chemistry, vol. 267, no. 28, pp. 2004420050, 1992.

[47] F. G. M. Snijdewint, P. Kaliński, E. A. Wierenga, J. D. Bos, and M. L. Kapsenberg, "Prostaglandin E2 differentially modulates cytokine secretion profiles of human T helper lymphocytes," Journal of Immunology, vol. 150, no. 12, pp. 5321-5329, 1993.

[48] A. J. Snider, "Sphingosine kinase and sphingosine-1-phosphate: regulators in autoimmune and inflammatory disease," International Journal of Clinical Rheumatology, vol. 8, no. 4, pp. 453463, 2013.

[49] E. Degagné and J. D. Saba, "S1 pping fire: sphingosine-1phosphate signaling as an emerging target in inflammatory bowel disease and colitis-associated cancer," Clinical and Experimental Gastroenterology, vol. 7, no. 1, pp. 205-214, 2014.
[50] Y. Deguchi, A. Andoh, Y. Yagi et al., "The S1P receptor modulator FTY720 prevents the development of experimental colitis in mice," Oncology Reports, vol. 16, no. 4, pp. 699-703, 2006.

[51] Y. Sanada, T. Mizushima, Y. Kai et al., "Therapeutic effects of novel sphingosine-1-phosphate receptor agonist W-061 in murine DSS colitis," PLoS ONE, vol. 6, no. 9, Article ID e23933, 2011.

[52] T. Mizushima, T. Ito, D. Kishi et al., "Therapeutic effects of a new lymphocyte homing reagent FTY720 in interleukin-10 genedeficient mice with colitis," Inflammatory Bowel Diseases, vol. 10, no. 3, pp. 182-192, 2004.

[53] J. Song, C. Matsuda, Y. Kai et al., "A novel sphingosine 1phosphate receptor agonist, 2-amino-2-propanediol hydrochloride (KRP-203), regulates chronic colitis in interleukin-10 genedeficient mice," Journal of Pharmacology and Experimental Therapeutics, vol. 324, no. 1, pp. 276-283, 2008.

[54] A. J. Snider, T. Kawamori, S. G. Bradshaw et al., "A role for sphingosine kinase 1 in dextran sulfate sodium-induced colitis," The FASEB Journal, vol. 23, no. 1, pp. 143-152, 2009.

[55] L. W. Maines, L. R. Fitzpatrick, K. J. French et al., "Suppression of ulcerative colitis in mice by orally available inhibitors of sphingosine kinase," Digestive Diseases and Sciences, vol. 53, no. 4, pp. 997-1012, 2008.

[56] J. Dong, H. Wang, G. Wu et al., "Oral treatment with SEW2871, a sphingosine-1-phosphate type 1 receptor agonist, ameliorates experimental colitis in interleukin-10 gene deficient mice," Clinical and Experimental Immunology, vol. 177, no. 1, pp. 94101, 2014.

[57] G. Giorgetti, G. Brandimarte, F. Fabiocchi et al., "Interactions between innate immunity, microbiota, and probiotics," Journal of Immunology Research, vol. 2015, Article ID 501361, 7 pages, 2015.

[58] M. J. Saez-Lara, C. Gomez-Llorente, J. Plaza-Diaz, and A. Gil, "The role of probiotic lactic acid bacteria and bifidobacteria in the prevention and treatment of inflammatory bowel disease and other related diseases: a systematic review of randomized human clinical trials," BioMed Research International, vol. 2015, Article ID 505878, 15 pages, 2015.

[59] Properties of Probiotics in Food including Powder Milk with Live Lactic Acid Bacteria, 2001, http://www.fao.org/3/a-a0512e .pdf.

[60] J. Ezendam and H. Van Loveren, "Probiotics: immunomodulation and evaluation of safety and efficacy," Nutrition Reviews, vol. 64, no. 1, pp. 1-14, 2006.

[61] C. M. Galdeano, A. De Moreno De Leblanc, G. Vinderola, M. E. Bibas Bonet, and G. Perdigón, "Proposed model: mechanisms of immunomodulation induced by probiotic bacteria," Clinical and Vaccine Immunology, vol. 14, no. 5, pp. 485-492, 2007.

[62] J. K. Limdi, C. O’Neill, and J. McLaughlin, "Do probiotics have a therapeutic role in gastroenterology?" World Journal of Gastroenterology, vol. 12, no. 34, pp. 5447-5457, 2006.

[63] R. A. Kekkonen, M. Sysi-Aho, T. Seppänen-Laakso et al., "Effect of probiotic Lactobacillus rhamnosus GG intervention on global serum lipidomics profiles in healthy adults," World Journal of Gastroenterology, vol. 14, no. 20, pp. 3188-3194, 2008.

[64] K. L. Madsen, "The use of probiotics in gastrointestinal disease," Canadian Journal of Gastroenterology, vol. 15, no. 12, pp. 817822, 2001.

[65] I. Soo, K. L. Madsen, Q. Tejpar et al., "VSL\#3 probiotic upregulates intestinal mucosal alkaline sphingomyelinase and reduces inflammation," Canadian Journal of Gastroenterology, vol. 22, no. 3, pp. 237-242, 2008. 
[66] S. Angulo, A. Morales, S. Danese et al., "Probiotic sonicates selectively induce mucosal immune cells apoptosis through ceramide generation via neutral sphingomyelinase," PLoS ONE, vol. 6, no. 3, Article ID e16953, 2011.

[67] Y. I. Kim, J. Y. Yang, H. J. Ko, M. N. Kweon, and S. Y. Chang, "Shigella flexneri inhibits intestinal inflammation by modulation of host sphingosine-1-phosphate in mice," Immune Network, vol. 14, no. 2, pp. 100-106, 2014.

[68] Z. Deng, J. Mu, M. Tseng et al., "Enterobacteria-secreted particles induce production of exosome-like S1P-containing particles by intestinal epithelium to drive Th17-mediated tumorigenesis," Nature Communications, vol. 6, article 6956, 2015.

[69] K. Yoshioka, Y. Ueno, S. Tanaka et al., "Role of natural killer $\mathrm{t}$ cells in the mouse colitis-associated colon cancer model," Scandinavian Journal of Immunology, vol. 75, no. 1, pp. 16-26, 2012.

[70] L. C. Wieland Brown, C. Penaranda, P. C. Kashyap et al., "Production of $\alpha$-galactosylceramide by a prominent member of the human gut microbiota," PLoS Biology, vol. 11, no. 7, article e1001610, 2013.

[71] D. An, S. F. Oh, T. Olszak et al., "Sphingolipids from a symbiotic microbe regulate homeostasis of host intestinal natural killer $\mathrm{T}$ cells," Cell, vol. 156, no. 1-2, pp. 123-133, 2014.

[72] P. J. Brennan, R. V. V. Tatituri, C. Heiss et al., "Activation of iNKT cells by a distinct constituent of the endogenous glucosylceramide fraction," Proceedings of the National Academy of Sciences of the United States of America, vol. 111, no. 37, pp. 1343313438, 2014. 


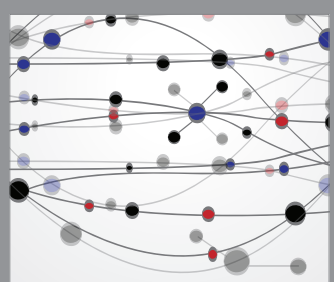

The Scientific World Journal
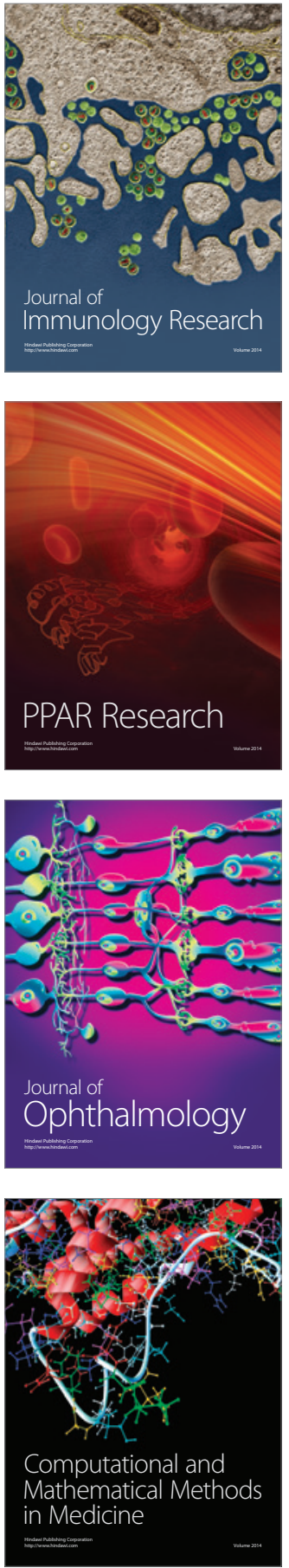

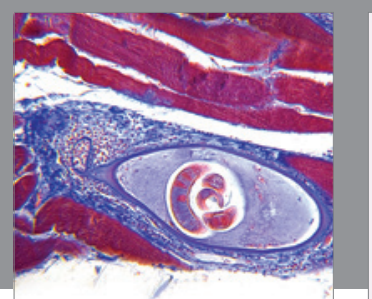

Gastroenterology Research and Practice

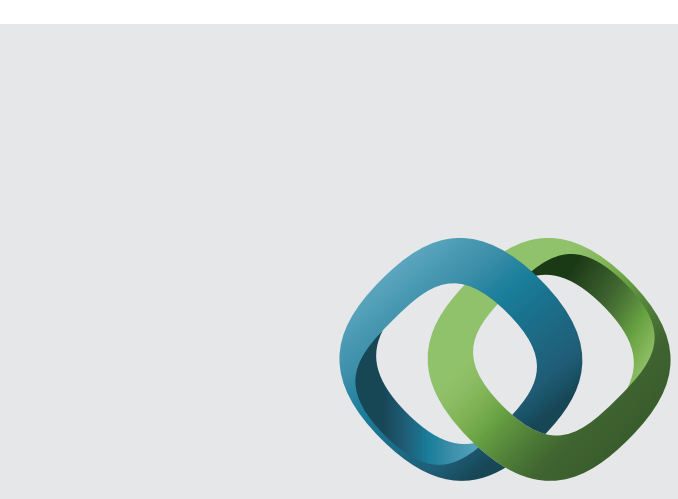

\section{Hindawi}

Submit your manuscripts at

http://www.hindawi.com
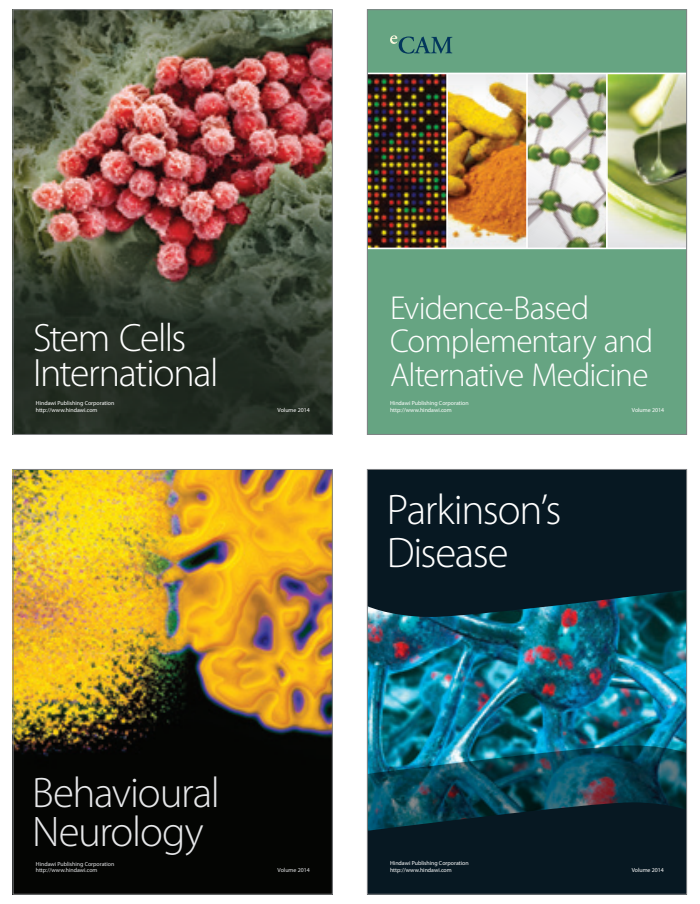
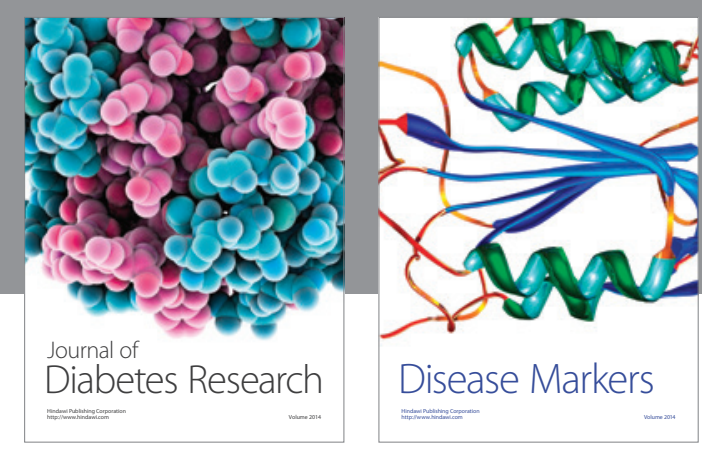

Disease Markers
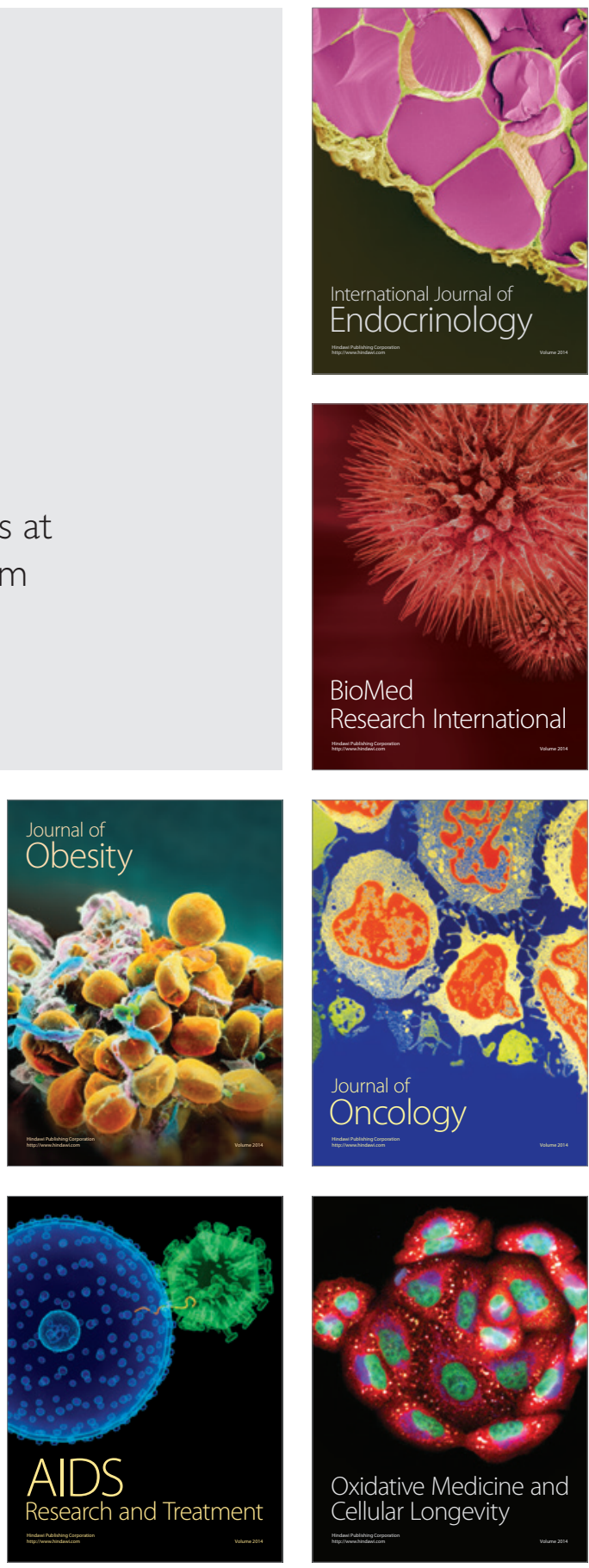